\title{
Gross Total Resection of Childhood Intracranial Ependymoma is a Critically Important Factor in Survival
}

\author{
(1) Rahmi Atıl AKSOY,' (1) Yasemin ŞENGÜN, ${ }^{1}$ (i) Melek Gamze AKSU, ${ }^{1}$ (i) Elif GÜLER, ${ }^{2}$ (i) Saim KAZAN, ${ }^{3}$ \\ (1) Mine GENÇ'
}

'Department of Radiation Oncology, Akdeniz University Faculty of Medicine, Antalya-Turkey ${ }^{2}$ Department of Pediatric Oncology, Akdeniz University Faculty of Medicine, Antalya-Turkey

${ }^{3}$ Department of Neurosurgery, Akdeniz University Faculty of Medicine, Antalya-Turkey

\section{OBJECTIVE}

This study aimed to evaluate the effect of prognostic factors and treatment on survival in childhood intracranial ependymoma.

\section{METHODS}

In the past two decades, 28 patients with pediatric intracranial ependymoma who received postoperative radiotherapy in our institute were evaluated statistically in terms of prognostic factors and survival. Progression-free survival (PFS) and overall survival (OS) were analyzed using the Kaplan-Meier method. Possible prognostic factors, such as sex, histopathological grade, the extent of resection, tumor location, spinal metastasis, and chemotherapy were also analyzed by log-rank test.

\section{RESULTS}

Median age at diagnosis was 4 years (range, 1-17 years). Eighteen patients had infratentorial tumor, and 19 patients had Grade III tumor. Gross total resections (GTRs) were performed in 16 patients. The median clinical follow-up time was 66.9 months (range, 8-253 months). The estimated 5-year PFS and OS rates are $38 \%$ and $55 \%$, respectively. The extent of resection was found the only prognostic factor associated with improved PFS and OS. Other factors, such as gender, histopathological grade, tumor location, spinal metastasis, and chemotherapy, showed no statistically significant effect on survival outcomes.

\section{CONCLUSION}

A multidisciplinary approach is required in the management of pediatric intracranial ependymomas. GTR is a crucial prognostic factor on survival. The lack of aggressive salvage treatments may result in worse survival. Future trials are needed to investigate molecular classification and individualized treatment algorithms.

Keywords: Ependymoma; intracranial; pediatric; prognosis.

Copyright $\odot$ 2022, Turkish Society for Radiation Oncology

\section{Introduction}

Ependymoma, the third most common pediatric central nervous system tumor following medulloblastoma and astrocytoma, originates from the walls of the ventricular system or the central canal of the spinal cord. $[1,2]$ Almost $90 \%$ of pediatric ependymomas are intracranial in origin and two-thirds are diagnosed in the 
posterior fossa.[3] Ependymomas represent approximately $10 \%$ of all childhood brain tumors and have a male-to-female ratio of 1.77:1.[4]

Based on the current WHO classification (2016), ependymal tumors can be classified as subependymoma (Grade I), myxopapillary ependymoma (Grade I), classic ependymoma (Grade II), anaplastic ependymoma (Grade III), and RELA-fusion-positive ependymoma (Grade II/III).[5] This classification scheme has limited clinical utility in predicting patients' results, therefore a modern molecular system has been suggested that separated intracranial ependymomas into six distinct subgroups. Supratentorial ependymomas were divided into three groups, such as YAP1, RELAfusion anaplastic ependymoma, and subependymoma. The three posterior fossa ependymoma (PF-EPN) subgroups were PF-EPN-A, PF-EPN-B, and PF-SE (subependymoma).[6]

A multidisciplinary approach is required in the management of pediatric intracranial ependymomas. Although surgery and post-operative radiotherapy (RT) are accepted standard of care for patients with non-disseminated ependymoma to reduce the risk of local recurrence, the role of chemotherapy in ependymoma remains unproven despite large clinical trials. [7] A post-operative magnetic resonance imaging (MRI) at no more than 3 days after surgery is indicated to detect extent of resection. A lumbar puncture performed at least 14 days postoperatively to exclude false-positive results, will determine spinal metastasis and guide management.[8] Craniospinal RT is required in the presence of spinal metastasis.[9]

The purpose of this study was to assess the effect of prognostic factors and treatment on progression-free survival (PFS) and overall survival (OS) in childhood intracranial ependymoma.

\section{Materials and Methods}

This retrospective study was undertaken after approval from our institutional ethics committee. A total of 28 patients, 15 males and 13 females, who received postoperative RT due to pediatric intracranial ependymoma between 2000 and 2020, were analyzed in this study. Inclusion criteria were patient age younger than 18 at the time of diagnosis, patients with confirmed histopathological diagnosis of Grade II/III intracranial ependymoma, and patients with no previous history of brain irradiation. Grade I ependymomas, spinal ependymomas, patients with severe comorbid disease, and patients with incomplete medical charts were not included in the study.

We evaluated patients' medical records, such as patient demographics, clinical procedures, localization of the tumor identified by neuroimaging, operation type, histopathologic results, and treatment modalities (i.e., RT and chemotherapy) applied postoperatively. The extent of surgical resection was determined based on the surgeon's operative report and/or post-operative MRI as gross total resection (GTR) or subtotal resection (STR). Spinal MRI and lumbar puncture were done in the post-operative period to reveal the presence of spinal seeding metastasis.

Radiation therapy was applied to all patients. Craniospinal RT was administered to patients with spinal seeding metastasis. Patients received conformal RT or intensity-modulated RT with a linear accelerator device. During RT planning, gross tumor volume was created by cross-section drawing in contouring tomography through pre-operative and post-operative MRI. Clinical target volume (CTV) was created by giving $0.5-1 \mathrm{~cm}$ margin to tumor bed, and planning target volume (PTV) was created by giving $0.3-0.5 \mathrm{~cm}$ margin to CTV. During the planning, when critical levels of organs at risk volumes were exceeded, manual corrections of PTV volume up to $0.3 \mathrm{~cm}$ were made.

All patients were evaluated clinically and radiographically at routine follow-up intervals. Patients who did not visit hospital for follow-up were called through telephone at the data cutoff point to determine their final status. Progression was diagnosed by clinical assessment, neuroimaging, and pathology reports. PFS was defined as the duration between diagnosis and the first event of recurrence or tumor progression, and OS was defined as the duration between diagnosis and death or last known date of the patients' survival.

All statistical analyses were performed using IBM SPSS v23.0. Descriptive analyzes were used to classify patients. PFS and OS were determined using the KaplanMeier method. Possible prognostic factors, such as gender, histopathological grade, extent of resection, tumor location, spinal metastasis, and chemotherapy, were also analyzed. Log-rank test was used to identify predictors of survival. $\mathrm{P}<0.05$ was considered statistically significant.

\section{Results}

Patient characteristics are summarized in Table 1. Median age at diagnosis was 4 years (range, 1-17 years). Most of the patients $(92.9 \%, n=26)$ were 3 years of 


\begin{tabular}{|c|c|c|}
\hline Characteristic & Absolute (n) & Relative (\%) \\
\hline \multicolumn{3}{|l|}{ Sex } \\
\hline Female & 13 & 46.4 \\
\hline Male & 15 & 53.6 \\
\hline \multicolumn{3}{|l|}{ Age at diagnosis } \\
\hline$<3$ years & 2 & 7.1 \\
\hline$\geq 3$ years & 26 & 92.9 \\
\hline \multicolumn{3}{|l|}{ Tumor location } \\
\hline Supratentorial & 10 & 35.7 \\
\hline Infratentorial & 18 & 64.3 \\
\hline \multicolumn{3}{|c|}{ Histopathological grade } \\
\hline Grade II & 9 & 32.1 \\
\hline Grade III & 19 & 67.9 \\
\hline \multicolumn{3}{|l|}{ Spinal metastasis } \\
\hline Yes & 5 & 17.9 \\
\hline No & 23 & 82.1 \\
\hline
\end{tabular}

age or older, and 2 patients $(7.1 \%)$ were younger than 3 years of age at the time of diagnosis. Ten of the patients included in the study had supratentorial, and 18 patients had posterior fossa located ependymoma. In our series $32.1 \%$ of patients had Grade II histology, and $67.9 \%$ of patients had Grade III histology.

Treatment parameters for patients are summarized in Table 2. All children with intracranial ependymoma underwent surgical resection of the primary tumor. GTR was achieved in 16 (57.1\%), and STR in 12 (42.9\%)

Table 2 Treatment parameters for patients

\begin{tabular}{lcc} 
Parameter & Absolute (n) & Relative (\%) \\
\hline $\begin{array}{l}\text { Surgery } \\
\text { STR }\end{array}$ & 12 & \\
$\quad$ GTR & 16 & 42.9 \\
RT volume & & 57.1 \\
$\quad$ Local & 23 & \\
$\quad$ Craniospinal & 5 & 17.9 \\
RT technique & & \\
$\quad$ 2D-RT & 7 & 25 \\
$\quad$ 3D-RT & 11 & 39.2 \\
$\quad$ IMRT & 10 & 35.8 \\
Total RT dose to primary site & & \\
$\quad$ <54 Gy & 4 & 14.2 \\
54 Gy & 19 & 67.9 \\
$\quad>54$ Gy & 5 & 17.9 \\
Chemotherapy & & \\
$\quad$ Yes & 14 & 50 \\
No & 14 & 50 \\
\hline
\end{tabular}

STR: Subtotal resection; GTR: Gross total resection; RT: Radiotherapy; IMRT: Intensity-modulated RT patients. The median time from initial surgery to RT was 2.1 months (range, 1-35 months). Twenty-three patients received only cranial RT and five patients received craniospinal RT. All patients' median cranial dose was $54 \mathrm{~Gy}$ (range, 45-60 Gy), and spinal dose of five patients who received craniospinal RT was 36 Gy. Daily fractionation was used with a median dose of $1.8 \mathrm{~Gy}$ (range, 1.6-2.0 Gy). Fourteen patients (50\%) received chemotherapy either concurrently, adjuvantly, or both.

Eighteen (64.2\%) patients suffered disease progression. The median time from diagnosis to progression was 46.7 months (range, 8-253 months). The 3 and 5 -year PFS rates of patients were $66 \%$ and $38 \%$, respectively. Statistical analysis showed that only the extent of resection was associated with improved PFS. The 5 -year estimated PFS rates in GTR group and STR group were $57 \%$ and $16 \%$, respectively $(\mathrm{p}=0.04)$. The PFS curves for GTR and STR patients are shown in Figure 1. After progression was detected, only four patients underwent re-surgery and only two patients were re-irradiated in our study.

After a median follow-up of 66.9 months (range, 8-253 months), 10 (35.7\%) patients were alive with no evidence of disease; 2 (7.1\%) were alive with disease; and $16(57.2 \%)$ were dead. The 3 and 5-year OS rates of patients were $88 \%$ and $55 \%$, respectively. Statistical analysis showed that only the extent of resection was associated with improved OS. The 5-year estimated OS rates in GTR group and STR group were $78 \%$ and $27 \%$, respectively ( $\mathrm{p}=0.02)$. The OS curves for GTR and STR patients are shown in Figure 2.

Other factors, such as gender, histopathological grade, tumor location, spinal metastasis, and chemotherapy, showed no significant effect on PFS and OS outcomes. The results of statistical analysis of prognostic factors are summarized in Table 3. No serious acute or late radiation complication was reported in the study patients.

\section{Discussion}

Management of childhood intracranial ependymomas requires multidisciplinary treatment approaches, and maximum surgical resection followed by RT is accepted as the current treatment standard. This recommendation is based on historical studies showing better survival results in children receiving post-operative RT compared to surgery alone, and in GTR group compared to STR group.[10-12] In our study, 28 pediatric intracranial ependymoma cases treated in line with these suggestions were retrospectively evaluated. 


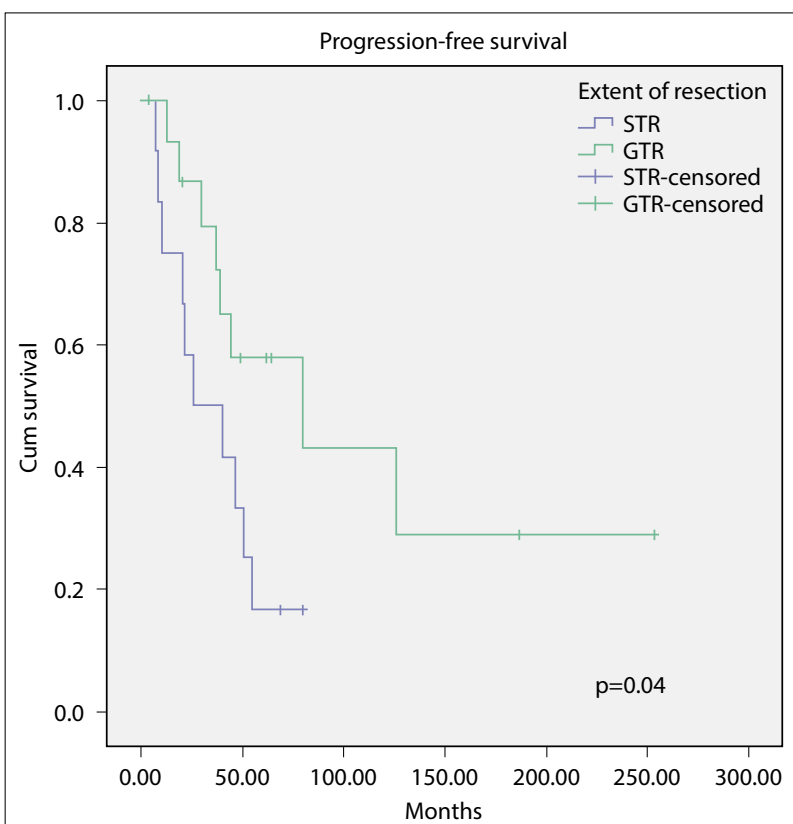

Fig. 1. Progression-free survival curves for all 28 patients according to the extent of resection. STR: Subtotal resection, GTR: Gross total resection.

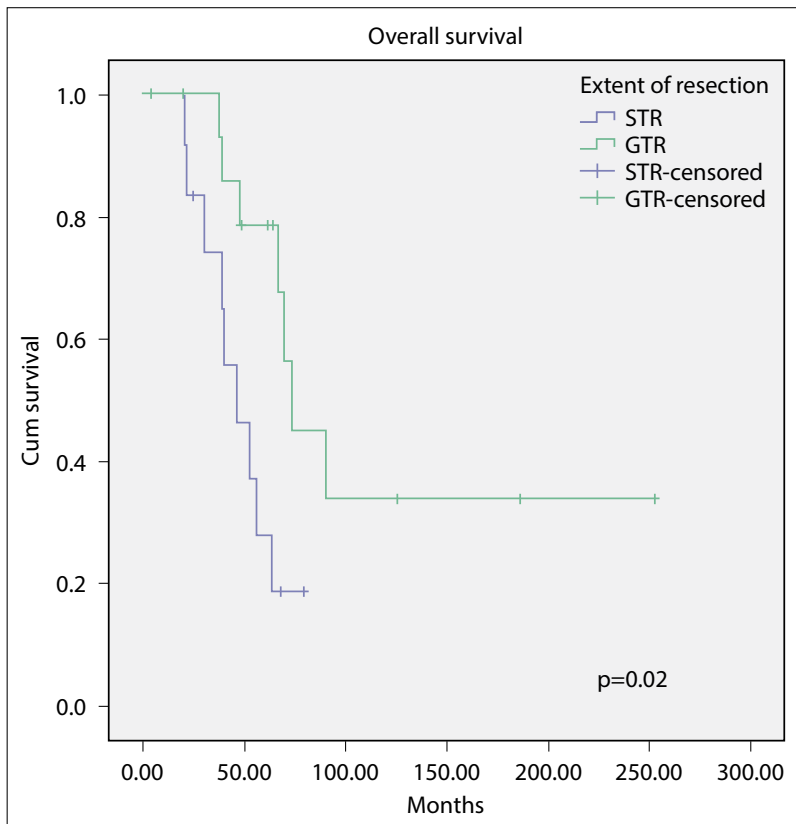

Fig. 2. Overall survival curves for all 28 patients according to the extent of resection.

STR: Subtotal resection, GTR: Gross total resection.

At present, it is estimated that complete resection is achieved in $70-90 \%$ of supratentorial ependymomas, but complete resection is less frequently possible in patients with infratentorial ependymomas which of- ten are located close to brainstem structures. Several studies confirmed the crucial role of a GTR in patients with newly diagnosed ependymomas.[13,14] However, the studies of Mansur et al. and Paulino et al.[15,16] did not find such a significant difference between total tumor removal and better survival.

Five-year PFS rates range from $50 \%$ to $70 \%$ after GTR and from zero to $30 \%$ after STR.[11,17] Similar to these reports, 5-year PFS rate was found as $57 \%$ in the GTR group, and $16 \%$ in the STR group, in our study $(\mathrm{p}=0.04)$. Five-year OS rates range from $80 \%$ to $90 \%$ after GTR and from $50 \%$ to $60 \%$ after STR.[14,18] In our study, 5-year OS rate was found as 78\% in the GTR group, and $27 \%$ in the STR group. Aggressive salvage local treatments for patients with residual disease can result in good OS. In the AIEOP study, 5 of the 17 patients with residual disease underwent re-surgery for potentially resectable tumor after chemotherapy and none of these operations were followed by persistent morbidity.[18] Similarly, Merchant et al.[14] have had some benefit with re-surgery and the second course of irradiation in selected patients. As local salvage therapy, only four patients underwent re-surgery and only two patients were re-irradiated in our study. The lack of aggressive salvage treatments may have caused our 5 -year OS rate in the STR group to remain lower than these studies. As a result of our study, it can be suggested that salvage treatments such as second-look surgery and re-irradiation should be improved in our institute.

The role of standard histologic classification in prognosis has been controversial. The second prospective AIEOP study which was stratified patients to histopathologic grade and extent of resection reported that higher 5-year PFS and OS rates among Grade II tumors (75.3\% and $90.5 \%)$, compared with Grade III tumors $(57.0 \%$ and $73.3 \%)(p=0.018$ in PFS and $p=0.031$ in OS).[19]. However, Agaoglu et al.[20] did not show any significant difference in OS or PFS between the two histologic subtypes. Similarly, distinct histological grades demonstrated no statistically significant differences in PFS and OS rates in our study. This finding may have occurred due to the insufficient number of patients.

As a result of advances in genomic, transcriptomic and epigenomic profiling, different molecular subtypes have been determined even for ependymomas of similar histology. Molecular subtypes have unique clinical characteristics and provide insights into individual treatment.[21] ST-EPN-RELA, which accounts for more than $70 \%$ of supratentorial ependymomas, occurs generally in children and young adults, and is associated with a poor prognosis. Conversely, ST-EPN- 


\begin{tabular}{|c|c|c|c|c|c|}
\hline Variable & Patients (n) & 5-year PFS (\%) & $\mathbf{p}$ & 5-year OS (\%) & $\mathbf{p}$ \\
\hline \multicolumn{6}{|l|}{ Gender } \\
\hline Female & 13 & 41 & 0.87 & 58 & 0.93 \\
\hline Male & 15 & 36 & & 54 & \\
\hline \multicolumn{6}{|l|}{ Tumor location } \\
\hline Supratentorial & 10 & 45 & 0.63 & 63 & 0.23 \\
\hline Infratentorial & 18 & 33 & & 51 & \\
\hline \multicolumn{6}{|c|}{ Histopathological grade } \\
\hline Grade II & 9 & 53 & 0.13 & 53 & 0.44 \\
\hline Grade III & 19 & 29 & & 56 & \\
\hline \multicolumn{6}{|l|}{ Extent of resection } \\
\hline STR & 12 & 16 & 0.04 & 27 & 0.02 \\
\hline GTR & 16 & 57 & & 78 & \\
\hline \multicolumn{6}{|l|}{ Spinal metastasis } \\
\hline Yes & 5 & 20 & 0.38 & 20 & 0.34 \\
\hline No & 23 & 42 & & 59 & \\
\hline \multicolumn{6}{|l|}{ Chemotherapy } \\
\hline Yes & 14 & 29 & 0.33 & 49 & 0.62 \\
\hline No & 14 & 46 & & 61 & \\
\hline
\end{tabular}

PFS: Progression-free survival; OS: Overall survival; STR: Subtotal resection; GTR: Gross total resection

YAP1 tumor is only seen in very young children and has a better prognosis.[22] The most common and aggressive subgroup, posterior fossa ependymoma group A (PF-EPN-A), appears in young children. In contrast, posterior fossa ependymoma Group B (PF-EPN-B) are seen in older children and has favorable clinical results. [23] Since the tests required for molecular subtyping could not be done in our institute, analyzes involving molecular subtypes were not performed in our study. Considering that individualized treatments will come to the fore in the future, molecular subtyping should be made available in cancer treatment centers.

Several studies have related that patients with supratentorial ependymomas have indicated better prognosis compared with patients with infratentorial location. [24,25] However, our results and others have failed to demonstrate a significant difference between location of tumor and survival.[14,26] Similar to our study, Paulino et al. and Tashvighi et al.[16,27] did not find such a significant difference between gender and survival. Conversely, Merchant et al.[14] found worse PFS in male patients $(\mathrm{p}=0.04)$.

In our study, 28 patients with pediatric intracranial ependymoma retrospectively evaluated and 5-year PFS and OS rates were found $38 \%$ and $55 \%$, respectively. Marinoff et al.[28] retrospectively evaluated 103 patients with median follow-up time of 11 years. They reported that 5-year PFS and OS rates were 39\% and
$67 \%$ and 10 -year PFS and OS rates were $29 \%$ and $50 \%$, respectively. They concluded that current management is not satisfactory to maintain long-term control of pediatric intracranial ependymoma, and novel treatment strategies are required.

The common approach is to deliver 50.4-59.4 Gy to the tumor bed with a margin. Local control rates are superior in patients treated with high dose RT, and current strategies propose $59.4 \mathrm{~Gy}$ for the volume at highest risk for local tumor recurrence.[4] The second prospective AIEOP study evaluated that patients with residual tumor received chemotherapy, second-look surgery, and 59.4 Gy RT followed by an 8 Gy boost in two fractions. They reported that this management tended to improve the prognosis of patients with residual tumors. [19] Correspondingly, the current SIOP-EP-II trial appraises the effect of a hypofractionated boost, $8 \mathrm{~Gy}$ in two fractions, to quantitative residual disease after tumor bed RT in an attempt to improve local control in this subgroup of children with a poorer prognosis.[29]

The benefits of chemotherapy in patients with newly diagnosed non-metastatic ependymoma remain debate. In our study, no statistically significant benefit was observed in terms of OS and PFS in the chemotherapy group. For patients with ependymoma, chemotherapy for two cycles is used postoperatively to improve the ability to perform a second surgery in patients with an STR. ACNS0121 is the first prospective trial, to suggest 
the use of chemotherapy and second surgery before RT. However, the effect of chemotherapy on survival was not demonstrated in the ACNS0121 trial.[30] The COG trial (ACNS0831, NCT01096368) and the SIOP trial (EP-II, NCT02265770) were launched to shed light on the usefulness of adjuvant chemotherapy.[29,31] The ACNS0831 trial is primarily evaluating PFS and OS in children between 1 and 20 years of age with nonmetastatic newly diagnosed ependymoma treated with local RT alone versus local RT followed by four cycles of adjuvant combination chemotherapy with cisplatin, cyclophosphamide, etoposide, and vincristine.[31] The SIOP-EP-II trial is primarily assessing GTR rate, PFS and number of treatment responders. In the SIOP-EPII trial, patients with no evidence of residual disease are randomly distributed to receive 16 weeks of multiagent chemotherapy or observation after RT, while patients with residual disease receive pre-RT conventional chemotherapy with or without methotrexate and post-RT conventional chemotherapy. [29]

\section{Conclusion}

Post-operative RT is an efficient treatment for childhood intracranial ependymoma. GTR is essential for longer PFS and OS among pediatric patients diagnosed with intracranial ependymoma. Better management will undoubtedly depend on classification according to the molecular biology of the tumor and tailoring the treatment to the individual. Future ependymoma trials should consider molecular classification when determining treatment indications and patient management.

Acknowledgments: We gratefully thank Deniz Ozel from Akdeniz University for the statistical analysis used in this study.

Peer-review: Externally peer-reviewed.

Conflict of Interest: All authors declared no conflict of interest.

Ethics Committee Approval: The study was approved by the Akdeniz University Faculty of Medicine Clinical Research Ethics Committee (No: KAEK-95, Date: 05/02/2020).

Financial Support: This study has received no financial support.

Authorship contributions: Concept - R.A.A.; Design E.G., M.G.A.; Supervision - M.G.; Funding - M.G.; Materials - S.K.; Data collection and/or processing - Y.Ş.; Data analysis and/or interpretation - R.A.A.; Literature search R.A.A., M.G.A.; Writing - R.A.A.; Critical review - M.G.

\section{References}

1. Vitanza NA, Partap S. Pediatric ependymoma. J Child Neurol 2016;31(12):1354-66.

2. Neumann JE, Spohn M, Obrecht D, Mynarek M, Thomas C, Hasselblatt M, et al. Molecular characterization of histopathological ependymoma variants. Acta Neuropathol 2020;139(2):305-18.

3. Kilday JP, Rahman R, Dyer S, Ridley L, Lowe J, Coyle $\mathrm{B}$, et al. Pediatric ependymoma: Biological perspectives. Mol Cancer Res 2009;7(6):765-86.

4. Gerstner ER, Pajtler KW. Ependymoma. Semin Neurol 2018;38(1):104-11.

5. Louis DN, Perry A, Reifenberger G, von Deimling A, Figarella-Branger D, Cavenee WK, et al. The 2016 World Health Organization classification of tumors of the central nervous system: A summary. Acta Neuropathol 2016;131(6):803-20.

6. Pajtler KW, Witt H, Sill M, Jones DT, Hovestadt V, Kratochwil F, et al. Molecular classification of ependymal tumors across all CNS compartments, histopathological grades, and age groups. Cancer Cell 2015;27(5):728-43.

7. Pajtler KW, Mack SC, Ramaswamy V, Smith CA, Witt $\mathrm{H}$, Smith A, et al. The current consensus on the clinical management of intracranial ependymoma and its distinct molecular variants. Acta Neuropathol 2017;133(1):5-12.

8. Toescu SM, Aquilina K. Current and emerging methods of management of ependymoma. Curr Oncol Rep 2019;21(9):78.

9. Rudà R, Reifenberger G, Frappaz D, Pfister SM, Laprie A, Santarius T, et al. EANO guidelines for the diagnosis and treatment of ependymal tumors. Neuro Oncol 2018;20(4):445-56.

10. Rousseau P, Habrand JL, Sarrazin D, Kalifa C, Terrier-Lacombe MJ, Rekacewicz C, et al. Treatment of intracranial ependymomas of children: Review of a 15-year experience. Int J Radiat Oncol Biol Phys. 1994;28(2):381-6.

11. Pollack IF, Gerszten PC, Martinez AJ, Lo KH, Shultz $\mathrm{B}$, Albright $\mathrm{AL}$, et al. Intracranial ependymomas of childhood: Long-term outcome and prognostic factors. Neurosurgery 1995;37(4):655-66.

12. Perilongo G, Massimino M, Sotti G, Belfontali T, Masiero L, Rigobello L, et al. Analyses of prognostic factors in a retrospective review of 92 children with ependymoma: Italian pediatric neuro-oncology group. Med Pediatr Oncol. 1997;29(2):79-85.

13. Nazar GB, Hoffman HJ, Becker LE, Jenkin D, Humphreys RP, Hendrick EB. Infratentorial ependymomas in childhood: Prognostic factors and treatment. J Neurosurg 1990;72(3):408-17.

14. Merchant TE, Li C, Xiong X, Kun LE, Boop FA, San- 
ford RA. Conformal radiotherapy after surgery for paediatric ependymoma: A prospective study. Lancet Oncol 2009;10(3):258-66.

15. Mansur DB, Perry A, Rajaram V, Michalski JM, Park TS, Leonard JR, et al. Postoperative radiation therapy for grade II and III intracranial ependymoma. Int J Radiat Oncol Biol Phys 2005;61(2):387-91.

16. Paulino AC, Wen BC, Buatti JM, Hussey DH, Zhen WK, Mayr NA, et al. Intracranial ependymomas: An analysis of prognostic factors and patterns of failure. Am J Clin Oncol 2002;25(2):117-22.

17. Robertson PL, Zeltzer PM, Boyett JM, Rorke LB, Allen JC, Geyer JR, et al. Survival and prognostic factors following radiation therapy and chemotherapy for ependymomas in children: A report of the children's cancer group. J Neurosurg 1998;88(4):695-703.

18. Massimino M, Gandola L, Giangaspero F, Sandri A, Valagussa P, Perilongo G, et al. Hyperfractionated radiotherapy and chemotherapy for childhood ependymoma: Final results of the first prospective AIEOP (Associazione Italiana di Ematologia-Oncologia Pediatrica) study. Int J Radiat Oncol Biol Phys 2004;58(5):1336-45.

19. Massimino M, Miceli R, Giangaspero F, Boschetti L, Modena P, Antonelli M, et al. Final results of the second prospective AIEOP protocol for pediatric intracranial ependymoma. Neuro Oncol 2016;18(10):1451-60.

20. Agaoglu FY, Ayan I, Dizdar Y, Kebudi R, Gorgun O, Darendeliler E. Ependymal tumors in childhood. Pediatr Blood Cancer 2005;45(3):298-303.

21. Khatua S, Mangum R, Bertrand KC, Zaky W, McCall D, Mack SC. Pediatric ependymoma: Current treatment and newer therapeutic insights. Future Oncol 2018;14(30):3175-86.

22. Hübner JM, Kool M, Pfister SM, Pajtler KW. Epidemiology, molecular classification and WHO grading of ependymoma. J Neurosurg Sci 2018;62(1):46-50.

23. Mack SC, Pajtler KW, Chavez L, Okonechnikov K, Bertrand $\mathrm{KC}$, Wang $\mathrm{X}$, et al. Therapeutic targeting of ependymoma as informed by oncogenic enhancer profiling. Nature 2018;553(7686):101-5.
24. Vaidya K, Smee R, Williams JR. Prognostic factors and treatment options for paediatric ependymomas. J Clin Neurosci 2012;19(9):1228-35.

25. Grill J, Le Deley MC, Gambarelli D, Raquin MA, Couanet D, Pierre-Kahn A, et al. Postoperative chemotherapy without irradiation for ependymoma in children under 5 years of age: A multicenter trial of the French society of pediatric oncology. J Clin Oncol 2001;19(5):1288-96.

26. Hammad M, Hosny M, Khalil EM, Alfaar AS, Fawzy M. Pediatric ependymoma: A single-center experience from a developing country. Indian $\mathrm{J}$ Cancer 2021;58(3):378-86.

27. Tashvighi M, Mehrvar A, Hedayati Asl AA, Mehrvar N, Ghorbani R, Naderi A, et al. Treatment challenges and outcomes for pediatric intracranial ependymoma at a single institution in Iran. Pediatr Hematol Oncol 2018;35(1):60-75.

28. Marinoff AE, Ma C, Guo D, Snuderl M, Wright KD, Manley PE, et al. Rethinking childhood ependymoma: A retrospective, multi-center analysis reveals poor long-term overall survival. J Neurooncol 2017;135(1):201-11.

29. ClinicalTrials.gov: NCT02265770: An International Clinical Program for the Diagnosis and Treatment of Children with Ependymoma (SIOP-EP-II). Available at: https://clinicaltrials.gov/ct2/show/ NCT02265770?term $=$ nct02265770\&rank=1

30. Merchant TE, Bendel AE, Sabin ND, Burger PC, Shaw DW, Chang E, et al. Conformal radiation therapy for pediatric ependymoma, chemotherapy for incompletely resected ependymoma, and observation for completely resected, supratentorial ependymoma. J Clin Oncol 2019;37(12):974-83.

31. ClinicalTrials.gov: NCT01096368: Maintenance Chemotherapy or Observation Following Induction Chemotherapy and Radiation Therapy in Treating Younger Patients with Newly Diagnosed Ependymoma. Available at: https:/clinicaltrials.gov/ct2/ show/NCT01096368?term=nct01096368 \&rank=1. Accessed Nov 15, 2021. 